\title{
Accurate diagnostics for schistosomiasis: a new role for PCR?
}

This article was published in the following Dove Press journal:

Reports in Parasitology

17 August 2015

Number of times this article has been viewed

\author{
Clive Shiff \\ Harry W Feinstone Department \\ of Molecular Microbiology and \\ Immunology, Johns Hopkins \\ Bloomberg School of Public Health, \\ Baltimore, MD, USA
}

\begin{abstract}
When it is important to detect schistosome infection with high sensitivity, particularly where local elimination is planned, detection of parasite-specific DNA should be considered as part of the diagnosis. The process of molecular amplification need no longer be considered to be restricted to sophisticated laboratories, but can be used effectively with modern tools in district hospitals or rural clinics and can be adapted for urine if it is used as the DNA source. With schistosomiasis, observation of a typical egg in urine, stool, or biopsy is taken as evidence of infection; point-of-care (POC) tests based on detecting parasite-specific antigens on a simple matrix have made this simpler and effective particularly in rural settings, but like observation of eggs, these methods are liable to give false negative results and thus miss infected cases. These cases are a likely source of parasite eggs to continue the infection cycle. Thus, although convenient, POC tests are unsatisfactory for epidemiological studies and evaluation of mass treatment campaigns. Methods to detect species-specific DNA fragments in fresh urine or in residue after filtration have been described and shown to be more sensitive and specific than specimen-based, serological, or antigen capture diagnosis. Parasite-specific DNA fragments can also be amplified from blood and stool taken from people infected with any of the major schistosomes. However, the use of urine residue after filtering through filter paper as a source of parasite-specific DNA has greatly simplified the process. Urine can be filtered at the POC using disposable plasticware and then paper dried and placed in a plastic sleeve, which is a simple process. If dry, the product is stable and transport is simple and inexpensive, and DNA can be extracted and amplified in a nearby facility set up for the work. If not POC, this is close to POC and quite feasible and processing by PCR or loop-mediated isothermal amplification process will ensure the universal application of this technology.
\end{abstract}

Keywords: schistosome differential diagnosis, urine-based test, DNA fragments, PCR, LAMP

\section{Introduction}

Schistosomiasis has been targeted for control and, hopefully, elimination. But for these parasites, as well as others, the choice of diagnostics for the diseases caused by them often presents a conundrum. The most expedient diagnostic systems are often easy to implement, cheap, and have a rapid response, but they tend to generate false negatives due to low sensitivity. The most sensitive diagnostics are often technically demanding, somewhat complicated, and somewhat costly. While both categories of diagnostic tests have a place, when it comes to monitoring the impact of the later stages of control programs when parasite prevalence and intensity are greatly reduced, there is a critical need for high sensitivity. False negatives are a major hazard to effective control because in schistosomiasis, a huge amplification takes place in the snails, which needs
Correspondence: Clive Shiff

Johns Hopkins Bloomberg School of Public Health, 615 North Wolfe Street, Baltimore, MD 21205, USA

Tel +l 4l0955 1263

Email cshiffl@jhu.edu 
to be inhibited. So, to assess the impact of large scale and repeated mass drug administration to control or even eliminate the problems, it is not sufficient to rely on estimates, but accurate diagnostics are required to identify and eliminate the low-intensity and residual infections. ${ }^{1}$ Only a few parasites can be amplified by intramolluscan reproduction and transmitted to humans via water contact. A limited number of surviving cases can spread infection rapidly and may negate the success of control programs. ${ }^{2}$ This ability to expand rapidly from a limited source is an important issue for other chronic, long-lasting parasite infections as well. Diagnostic tests with poor sensitivity will likely miss many of the chronic, lowlevel infections in communities as well as patients with partial cures. While this may not be a particularly serious problem for the patient, it provides a false picture of the prevalence of infection and thus compromises the ultimate goal of control programs. There is a need to transition to tests with higher sensitivity and specificity and to bring these tests closer to the point-of-care (POC). Molecular-based tests that detect small quantities of parasite DNA are currently available and these have been or can be adapted for field use. ${ }^{3}$ Thus, DNA amplification can be considered as available in most areas of need for improved diagnosis. Most district hospitals and health centers can operate the thermocycler equipment when needed, whether it is isothermal or polythermal. As long as the material to be examined is easily available, stable, and inexpensive to obtain, there is little reason not to expand the concept of POC diagnostics and enable use of simple, noninvasive, easily transported methods to collect and prepare specimens at the POC, and put them to use. The purpose here is to consider the molecular approaches that can be implemented to bring parasite detection closer to the goal of POC diagnostics.

\section{The importance of accurate diagnosis for schistosomiasis}

Schistosomiasis is a long-lived, chronic, and highly debilitating disease that affects rural and some urban populations across much of Africa and regions in South America, the Caribbean, People's Republic of China, and Southeast Asia. The disease in humans arises from infection by five trematode species. Schistosoma mansoni, Schistosoma japonicum, and Schistosoma mekongi normally live in the mesenteric veins and deposit eggs in the mesenteric venules that drain the gut epithelium. These eggs are normally passed in the feces. There are two other species, namely, Schistosoma haematobium and Schistosoma intercalatum that normally reside in vesicular veins draining the bladder epithelium, and the eggs of these species appear in the urine of infected hosts. These various parasites have different pathologies, and it is important to distinguish between them.

Schistosomes were first described in the mid-19th century and during the initial period covering the discovery and pathology of these parasites, treatment was rigorous and toxic and was based mainly on antimony and arsenic as the active agents. Positive diagnosis was essential prior to treatment and laboratory examination of urine or feces was the prime method, although rectal biopsy was often resorted to when specimen examination was considered insensitive. ${ }^{4}$ Emphasis on the need for positive diagnosis relaxed in the 1980s with the introduction of an effective and safe drug, praziquantel. When the cost of praziquantel declined with the availability of legal generic formulations, wide-scale mass treatment was commenced in several endemic areas to reduce the prevalence of the parasite, and the need for simple inexpensive measures of infection were sought to evaluate programs.

\section{Current diagnostic procedures with excreta}

Testing is simple for urinary schistosomiasis as hematuria in children has been used as a surrogate for the presence of adult worms in the bladder. ${ }^{5}$ Blood in urine, however, is not associated with the intestinal schistosomes, S. mansoni or S. japonicum, and diagnosis of these infections has depended on stool examination for the characteristic eggs of these parasites. ${ }^{6}$ This was initially done by simple filtration and sedimentation using 1-2 $\mathrm{g}$ of fresh stool and hand-held magnifying glass to observe any miracidium activity after the addition of clean water, ${ }^{7}$ or examination of stool debris on a microscope slide for the presence of eggs. ${ }^{8}$ The KatoKatz semiquantitative method is essentially performed on a stool smear. It is a direct examination of a measured $\sim 50 \mathrm{mg}$ of stool under a coverslip of glycerin-treated cellophane, using the microscope by a trained technician. This was introduced in the 1970s with some enthusiasm because of the added benefit of quantification. One can estimate the number of eggs per gram of feces and by inference, the intensity of infection. The Kato-Katz method has evolved as the gold standard for diagnosis of S. mansoni and S. japonicum and has been unofficially promoted by WHO through making the supplies for this assay available to field personnel. ${ }^{9}$ But this test determines the egg load in only a small subsample of the fecal specimen, making the test very low in sensitivity and certainly not a POC test. ${ }^{9}$ Still, the standard requirement was the detection of parasite eggs in urine or fecal specimen preparations, and the test has been universally used. However, the test is 
unreliable for the detection of chronic infections among adults and in children who have been treated. ${ }^{10,11-13}$ This is because passage of schistosome eggs can be erratic particularly in long-standing infections where granulomata and hyperplasia lesions occur in tissues through which eggs must pass to find routes to the exterior. In the case of urinary schistosomiasis, egg passage fluctuates diurnally and so depends on the time of day when the specimen is collected. ${ }^{14}$ However, these egg detection tests are still accepted as the reference - "(gold) standard" - by many workers. If the reference test is unreliable, how can it possibly be a "gold standard"? There needs to be a parasite-specific target that is indicative of the presence of a viable infection that is also readily available in blood, urine, or stool of the host.

\section{Antigen detection tests}

Pioneering work in this area was done by De Jonge et al from Holland, who detected specific schistosome antigens in blood and urine more than 50 years ago. ${ }^{15}$ This was done at a time when serology was in its early development and the structure of antibodies was being elicited. The conventional wisdom based on the then-current evidence was that antibodies did not develop fully for several years after birth and persisted for some time after the parasite was eliminated. Thus, they were of no diagnostic use in young children and yielded false positive results after curative treatment. De Jonge et al proposed that an improved and more specific test would be based on detection of some worm product in the form of a specific antigen that originated from viable parasites. ${ }^{15}$ Several of these antigens have been developed into POC diagnostics, but the most effective is the circulating cathodic antigen (CCA). This antigen as well as the circulating anodic antigen (CAA) were developed in Deelder's laboratory. ${ }^{15}$ These antigens were not properly defined, but apart from possessing different charges, the CCA was detectable in urine while the CAA was detectable in serum. ${ }^{16}$ Although the antigens were reported as "genus-specific", the CCA test now forms the basis of a commercial POC test that is widely used for $S$. mansoni but performs less effectively for S. haematobium. ${ }^{17,18}$ Diagnoses based on the detection of the CCA in urine are used because of convenience, ${ }^{18,19}$ but lack sensitivity in low-intensity infections such as in adults or children who have surviving parasites following ineffective treatment. ${ }^{20,21}$ This is critical when attempts to interrupt transmission are undertaken. ${ }^{2}$ Scientific advances are attempting to solve this problem and are currently following two routes: one based on detection of species-specific DNA in urine and the second using gene products or mass spectrography to identify specific proteins that can form the basis of more sensitive and specific antibody tests.

\section{DNA detection tests}

The concept of detecting schistosome DNA in a host was initiated by Hamburger et al when he showed that schistosome genome contained a high proportion of tandem repeat fragments that were recognized by specific probes in S. mansoni worms. ${ }^{22}$ He later showed that a similar fragment DRA-1 from $S$. haematobium could be used to detect developing schistosomes in the snail hosts, suggesting this may be of epidemiological importance. ${ }^{23}$ His group also demonstrated that his probe differentiated between $S$. haematobium and other non-human schistosome parasites in the snails. ${ }^{24}$ This DNA was detected in the tissue of snails and clearly demonstrated that the presence of the live parasite produced fragments of specific DNA and could be useful for accurate diagnosis. Latterly, that concept was used to detect parasites either in blood or in excreta, where the eggs or larvae might be found, and was shown to be more efficient than detection by microscopy. ${ }^{25}$ There is an alternative source of diagnostic DNA that is detectable in body fluids. Somatic DNA resulting from trophic activity of parasites or from products of disintegrating parasites is recognizable in serum or other body fluids. The rationale for this somewhat fragmented DNA was that parasites sloughed nuclear material into the blood, which subsequently crossed the transrenal barrier and passed into the urine or secreted in saliva. ${ }^{26}$ Thus, parasitespecific DNA from Leishmania ${ }^{27}$ and Plasmodium ${ }^{28}$ had been detected from either urine or saliva. Both S. haematobium and $S$. mansoni were also shown to excrete species specific DNA in the host urine, indicating the value of this route as evidence of infection. As a proof of the active nature of the indicator, it has also been shown that with $S$. haematobium, the DRA-1 fragment becomes undetectable 2 weeks following the treatment of the host. ${ }^{30,31}$ In addition, it has been shown that it is possible to differentiate between both schistosome species in a single urine specimen. ${ }^{32}$

\section{DNA detection improves sensitivity}

Strong evidence from numerous sources indicates that relying on the detection of schistosome eggs in urine or fecal specimens will miss a large proportion of infected individuals. ${ }^{33}$ This has been shown in two substantial studies of $S$. japonicum infections. First, a study by Xu et al was done in People's Republic of China. ${ }^{34}$ They measured a sixfold increase of prevalence using DNA-based detection in serum when compared with the cumulative result of nine successive 
Kato-Katz fecal examinations from each participant. The second study in the Philippines showed an increase in prevalence of S. japonicum from $22.9 \%$ (using Kato-Katz method) to $90.2 \%$ by performing real time polymerase chain reaction (RT-PCR) on stool specimen. ${ }^{35}$ In Zambia, Lodh et al showed that PCR detection of $S$. mansoni-specific DNA in urine residue on filter paper yielded a prevalence of $89 \%$ in a heterogeneous group of people, whereas Kato-Katz technique detected $51 \%$ and CCA test detected $60 \%$ of the infections. ${ }^{20}$ In work with urine-based DNA detection in Nigeria, Ibironke et al found that of a cohort of 89 adults, $64.0 \%$ were found to carry parasite-specific DNA in urine, while eggs were found in only $48 \%$ of the cases. ${ }^{29}$ Recent work from Kenya has demonstrated a lack of sensitivity in egg detection by Kato-Katz when compared with CCA test. ${ }^{31}$

In view of these discrepancies, why do current reviews discount such information and are not even mentioned in a Cochrane review? ${ }^{13,19}$ Clearly, the POC concept and tests mentioned earlier are extremely useful in assessing the local and school-wide extent of the problem, but this does not obviate the inherent weakness of the test. In schistosomiasis, the CCA-POC test is only effective for S. mansoni and performs inadequately for $S$. haematobium and $S$. japonicum. In addition, with $S$. mansoni, the test missed $33 \%$ of cases compared with the detection of DNA in urine. ${ }^{20}$ Yet, current reviews have discounted detection of parasite-specific DNA, be it in urine, stool, or blood, as too sophisticated for use in rural areas. ${ }^{13} \mathrm{~A}$ similar situation is happening now with malaria diagnostics. The malaria rapid diagnostic test, because of its convenience, is widely used without acceding to its lack of sensitivity and inability to detect low-level parasitic infections consistently. ${ }^{36}$ Clearly, there is a role for a convenient and reasonable accurate diagnostic test, but it must be used with the knowledge that a large proportion of asymptomatic cases will be missed and that these are also a reservoir of gametocytes liable to sustain the transmission cycle. ${ }^{36}$ However, in the malaria community, there is concern about the value of POC tests in diagnosis, but solutions are being sought; the situation is similar with helminth infections, and as pointed out in this paper, solutions do exist and these must be applied. It is of epidemiological importance to find and treat people with low-level infections if control operations are to be effective and sustained. The solution lies with DNA amplification procedures. The solution is to see how amplification technology can be simplified and incorporated into local health centers to enable the expansion of the POC concept.

\section{Revisiting the POC concept}

POC diagnostics are best used at the clinic or health center for rapid diagnosis of infectious diseases and have flourished since the advent of clinical thermometer. More recently, the discovery of monoclonal antibodies and their use in binding to specific antigens or targets in the organism of interest have expanded their applications. These operate best when used effectively by trained nurses and field technicians, and the results obtained for public health have been revolutionary. ${ }^{37}$ As such, they have proliferated in modern times and now cover several infections, even in home pregnancy, and other tests. The essential value of the POC test is that it is simple and inexpensive and has a reasonable sensitivity and specificity. It is useful for rapid diagnosis when treatment is locally available, for assessing outbreaks of infectious diseases, and, most essentially, is easily interpretable. We are considering the value of the test for schistosomiasis. For these parasites, POC tests are useful for one of the three main human parasites, ie, S. mansoni and can be used for epidemiological and prevalence studies that can serve as an affirmative diagnosis in the rural clinics. ${ }^{38}$ As primary diagnostic tests, they not only ensure that the drugs are given appropriately but also constitute the basis for targeted mass drug treatment to eliminate schistosomiasis foci in endemic areas. They can also be used to evaluate the treatment programs to reduce the overall prevalence of the infection.

POC tests have helped support the strategy for the progressive control of schistosomiasis in some endemic countries when it is based on systematic treatment of children at primary schools. ${ }^{39}$ This age group is most vulnerable to infections and disease, and is also important epidemiologically. ${ }^{40}$ Effective treatment of such vulnerable populations should eliminate a large proportion of the transmission and decrease the risk of infection. ${ }^{41}$ However, if a POC test is used for evaluation, it does beg the question about appropriate diagnostics, that of convenience and accuracy. The question that still remains unanswered is: "Where is the surviving reservoir of parasites?" Adults and untreated children continue to pass out limited numbers of eggs, and thus constitute a residual source of miracidia that can infect snails and maintain the cycle. These long-lived parasites are well adapted to our lifestyle and are also the source of chronic disease such as bladder cancer caused by S. haematobium in Egypt,${ }^{42}$ Ghana,${ }^{43}$ and Zimbabwe. ${ }^{44}$

There are several reasons to be concerned with "light" or chronic infections. First, there are post-treatment residual infections that persist in a community even after treatment 
with praziquantel. ${ }^{45}$ Second, in high transmission areas where people are exposed to cercariae, the schistosomula stage is not susceptible to praziquantel until it is fully transformed into an adult; so following treatment, there can be a type of recrudescence of the infection. ${ }^{46}$ Third, use of serology is often misleading, although dormant cases could be detected. However, serological tests are complex and not "POC" tests; they are also prone to give false positive results due to longlasting antibody reactions to infections. The problem as to how to detect residual or active infections in a simple and noninvasive manner raises the question: How can we bring DNA amplification procedures close to POC? This will in turn depend on the actual specimen provided by the patient.

Parasite-specific DNA can be detected in blood or urine, and in schistosomiasis the need for a stool specimen can be avoided. Urine is readily available, noninvasive, and can easily be collected at schools and clinics. Blood is also readily available as the patient is often willing to provide a small amount of blood for examination. Stool, however, is not always readily available and the specimen is noxious and can be infectious, thus requiring a protective environment for examination. Additionally, collecting a stool specimen entails an overnight stay on the part of the team, first to meet each person and offer a cup and spatula and then a second visit the next day to collect the specimen, package the contents, and cart off the collection boxes to a nearby or distant laboratory. There is increased expense involved in this. Procedures involved with dealing with stool collection and examination can be considered as POC diagnosis. Yet gathering a urine sample does offer simplicity and convenience. Both Ibironke et $\mathrm{al}^{29,30}$ and Lodh et $\mathrm{al}^{32}$ have shown that species-specific DNA of the parasite can be extracted and amplified from filter paper (Whatman number 3 ) through which approximately 50 $\mathrm{mL}$ urine is filtered. After filtration, the paper is dried under fly-proof cover, numbered, and packed with desiccant in a plastic sleeve. These sleeves can be easily transported, mailed, or sent with minimum expense to a laboratory either in the district or in some central facility for processing and examination. The DNA can be amplified by PCR and examined by electrophoresis or, as recently been shown, be amplified and visualized by the isothermal process known as loopmediated isothermal amplification process.$^{47}$ The process does not require a thermocycler or electrophoresis of the final product. It uses specialized primers and the process operates at one temperature defined by the primers, usually $65^{\circ} \mathrm{C}$, and hence can be done in a water bath or a battery-operated heating block if there is no electricity available. The product of amplification can be seen as a precipitate in the reaction tube. ${ }^{48}$ Alternatively, the product can be seen by adding a fluorescent product to the mix. ${ }^{49}$ This procedure can be carried out in a district or regional laboratory. Training and clean operation is required, but because the primers are specific for the target, amplification does not occur if the target is absent. Such a laboratory can be set up to service interventions where intervention operations are planned. The outlay and training required as well as the equipment and supplies are not expensive, but the results will be of considerable value to the success and sustainability of the interventions.

Thus, DNA amplification is a process that is close to being recognized as POC test. The analysis will be more sensitive than any other POC test, and the information relating to persons still found to be infected, and who should be provided with further treatment, will be available at short notice. The use of the ubiquitous cell phone helps with this. ${ }^{50}$ In this situation, the POC is expanded to involve simplicity of sample collection, use of simple equipment and supplies, and simplicity of operation to achieve accuracy of result. With rapid communication using mobile phones, the results can be provided with speed to any authorized body or person. ${ }^{50}$

\section{Conclusion}

Currently, the true POC tests for schistosomiasis are the antigen capture tests based on CCA or CAA. These have been examined in great detail (Ochodo et $\mathrm{al}^{19}$ ) and are deemed adequate for detection of $S$. mansoni infections but are less suitable for other schistosome species. ${ }^{51}$ However, the gold standard used for this Cochrane study was detection of parasite eggs in urine or fecal specimen preparations, and these have been shown to be quite unsatisfactory for the detection of infections among adults and in children who have been treated. ${ }^{10,11-13}$ This is because passage of schistosome eggs can be erratic, particularly in long-standing infections when granulomata and hyperplasia lesions occur in tissues through which eggs must pass to find routes to the exterior. Things can be improved if parasite-specific DNA detection is added, as suggested in this current study. Collection of urine specimens for this technique does not depend on the circadian rhythm; eggs are not the purpose of the specimen collection, it is a parasite DNA that has been filtered through the kidney, ${ }^{29}$ and collection of which does not pose a severe problem on any team that is participating in a mass control program. There is only one visit, as most people can produce a specimen on demand. The issue of expense has not been addressed in most studies that are associated with the current mass drug 
administration procedures, as most of these are part of ongoing studies to establish principles and practices. However, collection of stools in particular poses a high cost on diagnostics and personnel. Four-wheel drive trucks are expensive to use, but are essential to traverse rural roads in areas where schistosomes are endemic and usually require dedicated drivers. If urine is collected in rural schools, the process of filtration and drying can be overseen by a technician, but with the help from the school staff, the process of packing and transport to the nearest hospital or laboratory with loop-mediated isothermal amplification process facility will reduce costs considerably. If the mass drug administration program is to be integrated into a public health system and the need for accurate diagnosis is appreciated, the most convenient, rapid, and economical route is the urine specimen, ${ }^{30}$ and the DNA amplification is available in most endemic areas. All that is needed is a change in policy, local training, and some local infrastructure development.

\section{Acknowledgments}

I deeply acknowledge the support of my colleagues and coworkers from whom I have benefited over the years. First, I would like to honor late Dr Victor de V Clarke, Director of the Blair Research Laboratory, who was my friend for nearly 30 years, and from whom I learned the importance of accurate diagnosis of this serious and devastating parasite infection that ravaged the citizens of my home country. Thanks are also due to my students and colleagues at Johns Hopkins University, especially Alan Scott and David Sullivan who helped mold this paper.

\section{Disclosure}

The author reports no conflicts of interest in this work.

\section{References}

1. Shiff C. The importance of definitive diagnosis in chronic schistosomasis, with reference to Schistosoma haematobium. J Parasitol Res. 2012;2:761269.

2. Lelo AE, Mburu DN, Magoma GN, et al. No apparent reduction in schistosome burden or genetic diversity following four years of school-based mass drug administration in Mwea, Central Kenya, a heavy transmission area. PLoS Negl Trop Dis. 2014;8(10):e3221.

3. Tomita N, Mori Y, Kanda H, Notomi T. Loop-mediated isothermal amplification (LAMP) of gene sequences and simple visual detection of products. Nat Protocols. 2008;3(5):877-882.

4. Gelfand M. A Clinical Study of Intestinal Bilharziasis in Africa. London: Edwin Arnold; 1967.

5. Taylor PT, Murare HM, Manomano K. Efficacy of low doses of praziquantel for Schistosoma mansoni and S. haematobium. JTrop Med Hyg. 1988;91:13-17.

6. Colley DG, Bustinduy AL, Secor WE, King CH. Human schistosomiasis. Lancet. 2014;383(9936):2253-2264.

7. Clarke V. The influence of acquired resistance in the epidemiology of bilharziasis. Cent Afr J Med. 1966;12(Suppl 6):1-30.
8. Clarke V, Shiff CJ, Blair DM. The control of snail hosts of bilharziasis and fascioliasis in Southern Rhodesia. Bull World Health Organ. 1961;25:549-558.

9. Montresor A, Crompton D, Hall A, Bundy D, Savioli L. Guidelines for the Evaluation of Soil-Transmitted Helminths and Schistosomiasis at Community Level. Geneva: World Health Organization; 1998.

10. Garba A, Toure S, Dembele R, Bosque-Oliva E, Fenwick A. Implementation of national schistosomiasis control programmes in West Africa. Trends Parasitol. 2006;22(7):322-326.

11. Hotez PJ, Engels D, Fenwick A, Savioli L. Africa is desperate for praziquantel. Lancet. 2010;376(9740):496-498.

12. Colley DG, Binder S, Campbell C, et al. A five-country evaluation of a point-of-care circulating cathodic antigen urine assay for the prevalence of Schistosoma mansoni. Am J Trop Med Hyg. 2013; 88(3):426-432.

13. Stothard JR, Stanton M, Bustinduy AL, Sousa-Figueiredo JC, van Dam GJ, Betson M. Diagnostics for schistosomiasis in Africa and Arabia: a review of present options in control and future needs for elimination. Parasitology. 2014;141(Special Issue 14):1947-1961.

14. Weber MD, Blair DM, Clarke VV. The pattern of egg distribution in a micturition flow. Cent Afr J Med. 1967;13:75-88.

15. De Jonge N, Gryseels B, Hilberath GW, Polderman AM, Deelder AM. Detection of circulating anodic antigen by ELISA for seroepidemiology of schistosomiasis mansoni. Trans R Soc Trop Med Hyg. 1988;82(4):591-594.

16. Van Lieshout L, Polderman AM, Deelder AM. Immunodiagnosis of schistosomiasis by determination of the circulating antigens CAA and CCA, in particular in individuals with recent or light infections. Acta Trop. 2000;77(1):69-80.

17. Deelder AM, Qian ZL, Kremnser PG, et al. Quantitiative diagnosis of Schistosoma infections by measurement of circulating antigens in serum and urine. Trop Geogr Med. 1994;46:233-238.

18. Stothard JR, Sousa-Figueiredo JC, Standley C, et al. An evaluation of urine-CCA strip test and fingerprick blood SEA-ELISA for detection of urinary schistosomiasis in school children in Zanzibar. Acta Trop. 2009;111(1):64-70.

19. Ochodo E, Gopalakrishna G, Spek B, et al. Cochrane Database of Systematic Reviews. 2015;3. Art No CD009579.

20. Lodh N, Mwansa JC, Mutengo MM, Shiff CJ. Diagnosis of Schistosoma mansoni without the stool: comparison of three diagnostic tests to detect Schistosoma mansoni infection from filtered urine in Zambia. Am J Trop Med Hyg. 2013;89(1):46-50.

21. Koukounari A, Webster JP, Donnelly CA, et al. Sensitivities and specificities of diagnostic tests and infection prevalence of Schistosoma haematobium estimated from data on adults in villages northwest of Accra, Ghana. Am J Trop Med Hyg. 2009;80(3):435-441.

22. Hamburger J, Turetski T, Kapeller I, Deresiewicz R. The existance of highly repeated Highly repeated short DNA sequences in the genome of Schistosoma mansoni recognized by a species specific probe. Mol Biochem Parasitol. 1991;44:73-80.

23. Hamburger J, He N, Abbasi I, Ramzy RM, Jourdane J, Ruppel A. Polymerase chain reaction assay based on a highly repeated sequence of Schistosoma haematobium: a potential tool for monitoring schistosomeinfested water. Am J Trop Med Hyg. 2001;65(6):907-911.

24. Abbasi I, Hamburger J, Kariuki C, Mungai PL, Muchiri EM, King CH. Differentiating Schistosoma haematobium from related animal schistosomes by PCR amplifying inter-repeat sequences flanking newly selected repeated sequences. Am J Trop Med Hyg. 2012; 87(6):1059-1064.

25. NunezYO, Fernandez MA, Torres-Nunez D, et al. Multiplex polymerase chain reaction amplification and differentiation of Entamoeba histolytica and Entamoeba dispar DNA from stool samples. Am J Trop Med Hyg. 2001;64(5-6):293-297.

26. Mharakurwa S, Simoloka C, Thuma PE, Shiff CJ, Sullivan DJ. PCR detection of Plasmodium falciparum in human urine and saliva samples. Malar J. 2006;5:103.

27. Manna L, Reale S, Picillo E, Vitale F, Gravino A. Urine sampling for real-time polymeraase chain reaction-based diagnosis of canine leishmaniasis. J Vet Diagn Invest. 2008;20:64-67. 
28. Putaporntip C, Buppan P, Jongwutiwes S. Improved performance with saliva and urine as alternative DNA sources for malaria diagnosis by mitochondrial DNA-based PCR assays. Clin Microbiol Infect. 2011; 18(7):720.

29. Ibironke OA, Phillips AE, Garba A, Lamine SM, Shiff C. Diagnosis of Schistosoma haematobium by detection of specific DNA fragments from filtered urine samples. Am J Trop Med Hyg. 2011;84(6):998-1001.

30. Ibironke OA, Koukounari A, Asaolu S, Moustaki I, Shiff C. Validation of a new test for Schistosoma haematobium based on detection of the Dra1 DNA repeat fragment in urine: evaluation through latent class analysis. PLoS Negl Trop Dis. 2012;6(1):e1464.

31. Mwinzi PNM, Kittur N, Ochola E, et al. Additional evaluation of the point-of-contact circulating cathodic antigen assay for Schistosoma mansoni infection. Front Public Health. 2015;3:48.

32. Lodh N, Naples JM, Bosompem KM, Quartey J, Shiff CJ. Detection of parasite-specific DNA in urine sediment obtained by filtration differentiates between single and mixed infections of Schistosoma mansoni and S. haematobium from endemic areas in Ghana. PLoS One. 2014; 9(3):e91144.

33. Shiff C. New Diagnostics reform infectious parasite epidemiology. Lancet Infect Dis. 2014;14(6):446-448.

34. Xu X, Zhang Y, Lin D, et al. Serodiagnosis of Schistosoma japonicum infection: genome-wide identification of a protein biomarker, and assessment of its diagnostic validity in a field study in China. Lancet Infect Dis. 2014;14:489-497.

35. Gordon C, Acosta LP, Gobert GN, et al. Real-time PCR demonstrates high prevalence of Schistosoma japonicum in the Philippines: implications for surveillance and control. PLoS Negl Trop Dis. 2015; 9(1):e0003483.

36. Laban N, Kobayashi T, Hamapumbu H, et al. Comparison of a PfHRP2based rapid diagnostic test and PCR for malaria in a low prevalence setting in rural southern Zambia: implications for elimination. Malar J. 2015;14:25.

37. Stothard JR, Kabatereine NB, Tukahebwa EM, et al. Use of circulating cathodic antigen (CCA) dipsticks for detection of intestinal and urinary schistosomiasis. Acta Trop. 2006;97(2):219-228.

38. Sousa-Figueiredo JC, Betson M, Kabatereine NB, Stothard JR. The urine circulating cathodic antigen (CCA) dipstick: a valid substitute for microscopy for mapping and point-of-care diagnosis of intestinal schistosomiasis. PLoS Negl Trop Dis. 2013;7(1):e2008.

39. Fenwick A, Webster JP. Schistosomiasis: challenges for control, treatment and drug resistance [Miscellaneous]. Curr Opin Infect Dis. 2006 19(6):577-582.
40. Wilkins HA, Moore PJ. Comparative trials of regimes for the treatment of urinary schistosomiasis in the Gambia. J Trop Med Hyg. 1987; 90:83-92.

41. Kabatereine NB, Brooker S, Koukounari A, et al. Impact of a national helminth control programme on infection and morbidity in Ugandan schoolchildren. Bull World Health Organ. 2007;85(2):91-99.

42. Barsoum RS. Urinary schistosomiasis: a review. JAdv Res. 2013;4(5): 453-459.

43. Shiff CJ, Veltri R, Naples J, et al. Ultrasound verification of bladder damage associated with known biomarkers of bladder cancer in adults chronically infected with Schistosoma haematobium in Ghana. Trans $R$ Soc Trop Med Hyg. 2006;100:847-854.

44. Gelfand M, Weinberg RW, Castle WM. Relationship between carcinoma of the bladder and infestation with Schistosoma haematobium. Lancet. 1967;1967(i):1249-1251.

45. Lelo AE, Mburu DN, Magoma GN, et al. No apparent reduction in schistosome burden or genetic diversity following four years of schoolbased mass drug administration in MWEA, Central Kenya, a heavy transmission area. PLoS Negl Trop Dis. 2014;8(10):e3221.

46. Grandiere-Perez L, Ansart S, Paris L, Jaureguiberry S, Grivois J-P, Klement E. Efficacy of praziquantel during the incubation and invasive phase of Schistosoma haematobium schistosomiasis in 18 travellers. Am J Trop Med Hyg. 2006;74:814-818.

47. Hsiang MS, Greenhouse B, Rosenthal PJ. Point of care testing for malaria using LAMP, loop mediated isothermal amplification. J Infect Dis. 2014;210(8):1167-1169.

48. Mori Y, Nagamine K, Tomita N, Notomi T. Detection of loop-mediated isothermal amplification reaction by turbidity derived from magnesium pyrophosphate formation. Biochem Biophys Res Commun. 2001; 289(1):150-154.

49. Tomita N, Mori Y, Kanda H, Notomi T. Loop-mediated isothermal amplification (LAMP) of gene sequences and simple visual detection of products. Nat Protoc. 2008;3(5):877-882.

50. Kamanga A, Moono P, Stresman G, Mharakurwa S, Shiff C. Rural health centres, communities and malaria case detection in Zambia using mobile telephones: a means to detect potential reservoirs of infection in unstable transmission conditions. Malar J. 2010;9:96.

51. Ochodo EA, Spek B, Reitsma JB, et al. Rapid screening and diagnostic tests for human schistosomiasis in endemic areas (Protocol). Cochrane Database Syst Rev. 2012. doi:10.1002/14651858.CD009579.(2).
Reports in Parasitology

\section{Publish your work in this journal}

Reports in Parasitology is international, peer-reviewed, open access journal publishing original research, reports, reviews and commentaries on all areas of parasitology. The manuscript management system is completely online and includes a very quick and fair peer-review

\section{Dovepress}

system. Visit http://www.dovepress.com/testimonials.php to read real quotes from published authors. 